\title{
Chemical

\section{Structural Evidence of Perfluorooctane Sulfonate Transport by Human Serum Albumin}

\author{
Zhipu Luo, ${ }^{\dagger, \ddagger}$ Xiaoli Shi, ${ }^{\dagger}$ Qin $\mathrm{Hu}^{\S}{ }^{\S}$ Bin Zhao, ${ }^{\S}$ and Mingdong Huang ${ }^{*}, \dagger$ \\ ${ }^{\dagger}$ State Key laboratory of Structural Chemistry, Fujian Institute of Research on the Structure of Matter, Chinese Academy of Sciences, \\ Fuzhou 350002, China \\ ${ }^{\ddagger}$ Graduate University of the Chinese Academy of Sciences, Beijing 100049, China \\ ${ }^{\S}$ Research Center for Eco-Environmental Sciences, Chinese Academy of Sciences, Beijing 100085, China
}

\section{Supporting Information}

ABSTRACT: Perfluorooctane sulfonate (PFOS) is a man-made fluorosurfactant and globally persistent organic pollutant. PFOS is mainly distributed in blood with a long half-life for elimination. PFOS was found mainly bound to human serum albumin (HSA) in plasma, the most abundant protein in human blood plasma, which transports a variety of endogenous and exogenous ligands. However, the structural basis of such binding remains unclear. Here, we report the crystal structure of the HSA-PFOS complex and show that PFOS binds to HSA at a molar ratio of 2:1. In addition, PFOS binding renders the HSA structure more compact. Our results provide a structural mechanism to understand the retention of surfactants in human serum.

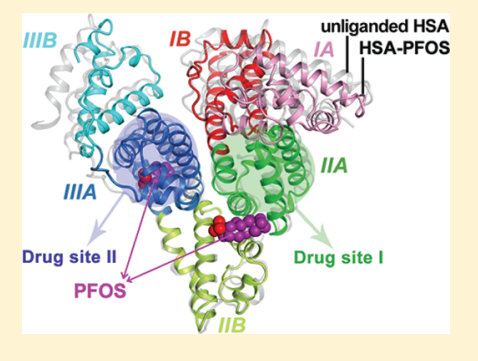

$\mathrm{P}$ erfluorooctane sulfonate (PFOS) (Supporting Information, Figure $\mathrm{S} 1$ ) is a stable chemical with high surface activity, high thermal and acid resistance, and with both hydroand lipophobic properties. PFOS have been used in a wide range of industrial and commercial applications since 1950s. ${ }^{1}$ PFOS has been found to cause toxicity to human health, including reproductive toxicity, neurotoxicity, and hepatotoxicity. ${ }^{2,3}$ Unlike most organic pollutants, such as dioxins, PFOS does not accumulate in fatty tissue but mainly in blood and liver, ${ }^{4-6}$ and has been detected in the blood of humans in many countries. $^{7,8}$ The half-life of elimination of PFOS in human serum was quite long (estimated about 5.4 years). ${ }^{9}$ In May 2009, PFOS was added into Annex B of the Stockholm Convention on Persistent Organic Pollutants for restrictions on production and use. ${ }^{10}$

Human serum albumin (HSA), produced in the liver, is the major soluble protein in human blood plasma with a concentration of about $0.64 \mathrm{mM}$ and has many physiological functions. ${ }^{11}$ Albumin maintains the colloid osmotic blood pressure and plays an important role in the transport, distribution, and metabolism of many endogenous and exogenous compounds, especially fatty acids (FA) and drugs. ${ }^{12}$ Drug binding to albumin can significantly alter the half-life, distribution, and metabolism of drugs, therefore playing a major role in the absorption, distribution, metabolism, and secretion of many important pharmaceuticals. ${ }^{13}$ Sudlow found two primary drug-binding sites on the protein, named Sudlow sites I and II. ${ }^{14,15}$ The first crystallographic structure of HSA showed that HSA is a heart-shaped protein, which is composed of 585 amino acid residues with three structurally homologous domains (I, II, and III). Each contains $10 \alpha$-helices that can be grouped into six-helix and four-helix subdomains (A and $\mathrm{B}$ ). The primary drug-binding sites I and II on HSA are located in subdomains IIA and IIIA, respectively (figure in Abstract). ${ }^{16}$ Subsequently, additional drug binding sites have also been found. ${ }^{17-20}$

As the most abundant protein in plasma, HSA has been shown to be the main carrier protein for PFOS. PFOS was demonstrated to bind strongly to bovine serum albumin in a 1:1 stoichiometric ratio. ${ }^{21}$ A fluorescence study of PFOS binding to HSA showed that PFOS can bind to HSA at Sudlow sites I and II, with a binding constant of $2.2 \times 10^{4} \mathrm{M}^{-1}$ at site I and $7.6 \times 10^{6} \mathrm{M}^{-1}$ at site II. ${ }^{22}$ The ratio of PFOS to HSA at saturation binding was determined to be $45: 1 .^{23}$ Molecular modeling was also used to study the binding sites of PFOS on HSA, which showed that the tight binding site is near Trp214 or Sudlow site I and that the maximum number of PFOS binding to HSA is $11 .^{24}$ However, the structural details of PFOS binding to HSA remain unclear. Here, we report the crystallization of the HSA-PFOS complex and its crystal structure at a resolution of $2.25 \AA$ (figure in Abstract and Supporting Information, Table 1). The structure of the complex shows two PFOS molecules bound to HSA, with one located at Sudlow site II (subdomain IIIA) and the other located at the interface of subdomains IIA and IIB. Here, we refer to these two PFOS molecules as PFOS3 and PFOS6, respectively, in the following structural analysis, using the numbering scheme of the HSA ligand binding site that was established by Stephen Curry. ${ }^{25,26}$ These PFOS molecules overlap with fatty acid binding sites FA3/4 and FA6, respectively (Figure $1 \mathrm{C}, \mathrm{D}$ ).

PFOS3 was located in Sudlow site II in subdomain IIIA, which typically contains two binding sites for fatty acid, i.e.,

Received: March 13, 2012

Published: April 6, 2012 


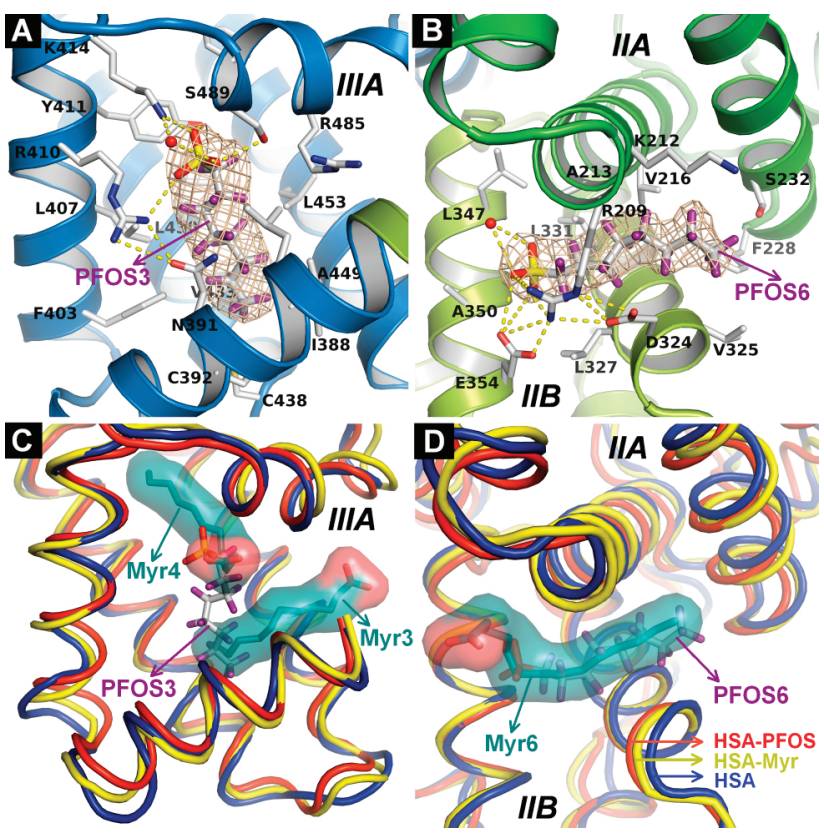

Figure 1. Binding of PFOS in HSA. (A and B) PFOS binding sites at subdomain IIIA (PFOS3) and the interface of subdomains IIA and IIB (PFOS6). Subdomains IA, IB, IIA, IIB, IIIA, and IIIB are colored pink, red, green, lemon, blue, and cyan, respectively. Electron density is in mesh presentations colored wheat. Atom colors: carbon, gray; nitrogen, blue; oxygen, red; fluorine, purple; sulfur, yellow. Hydrogen bonds are shown as yellow dashed lines. (C and D) Comparison of local conformational change on PFOS binding sites by superposing ligand-free HSA (PDB code 1UOR, colored blue) and HSA-myristate (Myr) (PDB code 1E7G, colored yellow) structure to the HSA-PFOS (colored red) structure. Myristates are shown as dark cyan sticks and surface.

FA3 and FA4. Myristate (Myr) was used for illustrating the FA binding sites. In our study, the PFOS3 molecule was found to stretch across both Myr3 and Myr4 binding sites (Figure 1C). The sulfonate headgroup of PFOS3 was fixed in the same position of the carboxylate head groups of Myr4 by a strong salt bridge to K414 and hydrogen bonding to R410 and Y411, while the trifluoromethyl tail of the PFOS3 aligned well to the methylene tail of fatty acid Myr3 typically bound at this position and interacted with the side chain of nonpolar amino acid (L387, I388, C392, F403, L407, L430, V433, C438, A449, and L453) through hydrophobic interaction (Figure 1A). These interactions of PFOS with HSA make subdomain IIIA more compact and leave no room for more PFOS molecules to occupy the remaining space of FA3/FA4 binding sites.

PFOS6 occupies the FA6 binding site, which is located at the interface of HSA subdomains IIA and IIB. The sulfonate headgroup of PFOS6 points to the same direction of the carboxylate head groups of Myr6 (Figure 1D) and interacts with polar residues (R209 and E354) by hydrogen bonds. The current structure has high resolution allowing the identification of water molecules around the sulfonate headgroup of PFOS6. The trifluoromethyl tail of PFOS6 interacted with the hydrophobic residues of HSA (V216, F228, V325, L327, and L331) (Figure 1B). Through these interactions, the local conformation has changed with helix h3 and helix h4 of subdomain IIA shifted toward subdomain IIB compared to that in the unliganded HSA or HSA-Myr complex domain II (Supporting Information Figure S2).
To further study the conformational change of HSA induced by PFOS, we compared the structures of unliganded HSA (PDB code 1UOR), HSA-Myr complex (PDB code 1EG7), and our HSA-PFOS complex. Since the domain IIB is most structurally conserved in HSA structures, we superposed the subdomain IIB domain of HSA and observed large domain shifts of subdomain IA and IIIB among these structures (Figure 2). Domains I and III of both the ligand-free HSA and HSA-

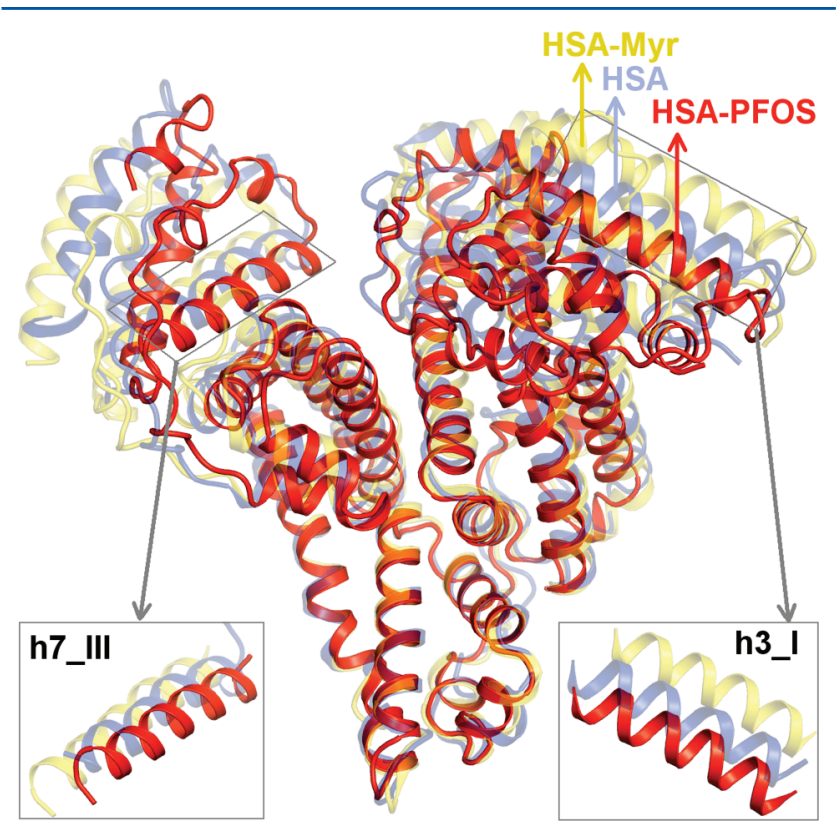

Figure 2. PFOS-induced structure compaction of HSA. HSA-PFOS complex (colored red) with aligned to unliganded HSA (PDB code 1UOR, colored blue) and HSA-Myr complex (PDB code 1E7G, colored yellow) by domain IIB. Helix h3 of domain I and Helix h7 of domain III were picked out to show the helix shift.

Myr complex shifted upward from subdomain IIB of the HSAPFOS complex, whereas the HSA-PFOS structure becomes more compact compared to the structures of unliganded HSA and the HSA-fatty acid complex. Helix h3 of domain I (residue 38-55) and helix h7 of domain III (residue 517-537) have the largest shifts of $\mathrm{C} \alpha$ atoms $(15.1 \AA$ and $7.8 \AA$ ) compared to that in the HSA-Myr complex and unliganded HSA. The solvent accessible surface area of HSA-PFOS is much smaller compared to that of the unliganded HSA and HSA-Myr complex (27119 $\AA^{2}$ vs $31660 \AA^{2}$ and $31929 \AA^{2}$ from residues 5 to 76 and from 89 to 571 without including ligands and water molecules). All these results suggest that PFOS binding renders HSA more compact. Such conformational changes are different from that induced by fatty acids that are well demonstrated. ${ }^{25,27}$

Furthermore, we used circular dichroism to analyze the thermal stability of the HSA-PFOS complex. The transformation temperature of the HSA-PFOS complex is $4{ }^{\circ} \mathrm{C}$ higher than that of HSA (Supporting Information, Figure S3), indicating that the PFOS binding increases albumin thermal stability. Previous studies using isothermal titration calorimetry showed that the interactions of PFOS with HSA were spontaneous and driven by entropy change, ${ }^{23}$ which indicated that the HSA-PFOS complex becomes less mobile compared to HSA without ligands.

Sudlow site $\mathrm{I}$ is an important drug binding site and was previouly shown to be the potential PFOS binding site. ${ }^{21,22,24}$ However, we did not find clear density for the PFOS molecule 
at this site in our experimental condition. One possible reason is that the perfluorinated carbon chain is structurally rigid due to the strong repulsive force between the fluorine atom (in contrast to the flexible acyl chain of fatty acids) and thus cannot be accommodated at this site which has a number of polar residues in the middle of the pocket. $^{28}$

In summary, our structural study of the HSA-PFOS complex reveals two PFOS binding sites on the protein, with one located at Sudlow site II and the other located at the FA6 binding site. The strongly polar sulfonyl group of PFOS interacts with the hydrophilic residues of HSA, while the perfluorinated carbon tail of PFOS interacts with the adjacent hydrophobic residues of HSA (Supporting Information, Figure S4). The overall structure of the HSA-PFOS complex becomes more compact than the unliganded HSA, which is quite different from the HSA-fatty acid complex. Such results may partially explain the long plasma half-life of PFOS (5.4 years). ${ }^{9}$ This study gives new structural insight into PFOS interaction with protein and also provides a structural basis for the design of a future generation of fluoro-surfactants.

\section{ASSOCIATED CONTENT}

\section{S Supporting Information}

Experimental procedures; X-ray data collection and refinement statistics for the HSA-PFOS complex structure; chemical structures of PFOS; domain movement near the PFOS6 binding site; CD unfolding of ligand-free HSA and HSA-PFOS complex; hydrophobility and hydrophility of PFOS binding sites. This material is available free of charge via the Internet at http://pubs.acs.org.

\section{Accession Codes}

The atomic coordinates and structure factors of the HSAPFOS complex have been deposited in the Protein Data Bank with the accession number 4E99.

\section{AUTHOR INFORMATION}

\section{Corresponding Author}

*Tel: (86) 591-83704996. Fax: (86) 591-83703926. E-mail: mhuang@fjirsm.ac.cn.

\section{Funding}

This work is supported by grants from Natural Science Foundation of China (30800181 and 21171167) and Ministry of Science of Technology of China (2007CB914304).

\section{Notes}

The authors declare no competing financial interest.

\section{ABBREVIATIONS}

FA, fatty acid; HSA, human serum albumin; Myr, myristate; PDB, protein data bank; PFOS, perfluorooctane sulfonate

\section{REFERENCES}

(1) 3M (1999) USEPA EPA-HQ-OPPT-2002-0043-0006.

(2) Genuis, S. J., Birkholz, D., Ralitsch, M., and Thibault, N. (2010)

Public Health 124, 367-375.

(3) OECD (2002) ENV/JM/RD(2002)17/FINAL, p 5.

(4) Kannan, K., Koistinen, J., Beckmen, K., Evans, T., Gorzelany, J. F., Hansen, K. J., Jones, P. D., Helle, E., Nyman, M., and Giesy, J. P. (2001) Environ. Sci. Technol. 35, 1593-1598.

(5) Martin, J. W., Mabury, S. A., Solomon, K. R., and Muir, D. C. (2003) Environ. Toxicol. Chem. 22, 196-204.

(6) Taniyasu, S., Kannan, K., Horii, Y., Hanari, N., and Yamashita, N. (2003) Environ. Sci. Technol. 37, 2634-2639.
(7) Kannan, K., Corsolini, S., Falandysz, J., Fillmann, G., Kumar, K. S., Loganathan, B. G., Mohd, M. A., Olivero, J., Van Wouwe, N., Yang, J. H., and Aldoust, K. M. (2004) Environ. Sci. Technol. 38, 4489-4495.

(8) Yeung, L. W., So, M. K., Jiang, G., Taniyasu, S., Yamashita, N., Song, M., Wu, Y., Li, J., Giesy, J. P., Guruge, K. S., and Lam, P. K. (2006) Environ. Sci. Technol. 40, 715-720.

(9) Olsen, G. W., Burris, J. M., Ehresman, D. J., Froehlich, J. W., Seacat, A. M., Butenhoff, J. L., and Zobel, L. R. (2007) Environ. Health Perspect. 115, 1298-1305.

(10) Wang, T., Wang, Y., Liao, C., Cai, Y., and Jiang, G. (2009) Environ. Sci. Technol. 43, 5171-5175.

(11) Peters, T. (1995) All About Albumin: Biochemistry, Genetics, and Medical Applications. Academic Press, New York.

(12) Kragh-Hansen, U. (1981) Pharmacol. Rev. 33, 17-53.

(13) Carter, D. C., and Abraham, D. J. (2003) Crystallographic Survey of Albumin Drug Interaction and Preliminary Applications in Cancer Chemotherapy, in Burger's Medicinal Chemistry and Drug Discovery, John Wiley \& Sons, Inc., New York.

(14) Sudlow, G., Birkett, D. J., and Wade, D. N. (1975) Mol. Pharmacol. 11, 824-832.

(15) Sudlow, G., Birkett, D. J., and Wade, D. N. (1976) Mol. Pharmacol. 12, 1052-1061.

(16) He, X. M., and Carter, D. C. (1992) Nature 358, 209-215.

(17) Ghuman, J., Zunszain, P. A., Petitpas, I., Bhattacharya, A. A., Otagiri, M., and Curry, S. (2005) J. Mol. Biol. 353, 38-52.

(18) Zhu, L., Yang, F., Chen, L., Meehan, E. J., and Huang, M. (2008) J. Struct. Biol. 162, 40-49.

(19) Zunszain, P. A., Ghuman, J., McDonagh, A. F., and Curry, S. (2008) J. Mol. Biol. 381, 394-406.

(20) Hein, K. L., Kragh-Hansen, U., Morth, J. P., Jeppesen, M. D., Otzen, D., Moller, J. V., and Nissen, P. (2010) J. Struct. Biol. 171, 353360.

(21) Jones, P. D., Hu, W., De Coen, W., Newsted, J. L., and Giesy, J. P. (2003) Environ. Toxicol. Chem. 22, 2639-2649.

(22) Chen, Y. M., and Guo, L. H. (2009) Arch. Toxicol. 83, 255-261.

(23) Zhang, X., Chen, L., Fei, X. C., Ma, Y. S., and Gao, H. W. (2009) BMc Mol. Biol. 10, 16.

(24) Salvalaglio, M., Muscionico, I., and Cavallotti, C. (2010) J. Phys. Chem. B 114, 14860-14874.

(25) Curry, S., Mandelkow, H., Brick, P., and Franks, N. (1998) Nat. Struct. Biol. 5, 827-835.

(26) Bhattacharya, A. A., Grune, T., and Curry, S. (2000) J. Mol. Biol. 303, 721-732.

(27) Fujiwara, S., and Amisaki, T. (2006) Proteins 64, 730-739.

(28) Curry, S. (2009) Drug Metab. Pharmacokinet. 24, 342-357. 